\title{
VALORES HEMATOLÓGICOS DEL MACHÍN NEGRO (Cebus apella) MANTENIDO EN CAUTIVERIO
}

\author{
Haematological Values in Tufted Capuchin (Cebus apella) Reared \\ in Captivity
}

Pedro A. Ospina S. ${ }^{1,2}$, Olga Li E. ${ }^{1,3}$, David Tribeño H. ${ }^{4}$ y Jacqueline Cahua U. ${ }^{5}$

\section{RESUMEN}

\begin{abstract}
El objetivo del presente estudio fue determinar los valores hematológicos referenciales del machín negro (Cebus apella) mantenidos en cautiverio en el Patronato del Parque de Las Leyendas, Lima. Se utilizaron 32 primates (25 machos y 7 hembras), entre juveniles, sub-adultos y adultos, en aparente buen estado de salud. Los primates fueron anestesiados con una combinación de ketamina $(10 \mathrm{mg} / \mathrm{kg} / \mathrm{pv})$ y xilazina $(1 \mathrm{mg} / \mathrm{kg} / \mathrm{pv})$, vía intramuscular, a través de una malla de contención. Se recolectó muestras de sangre por punción de la vena femoral utilizando vacutainers con anticoagulante EDTA. Se realizó el recuento de glóbulos rojos (GR) y blancos (GB) utilizando la cámara de Neubauer, el recuento plaquetario y diferencial utilizando la coloración de Wright, la determinación de la hemoglobina $(\mathrm{Hb})$ con el método de cianometahemoglobina, el hematocrito $(\mathrm{Ht})$ a través del microhematocrito y los índices eritrocíticos aplicando fórmulas estándares con los valores de GR, Hb y Ht. Los valores promedio calculados fueron de GR: 5.12 x 10\% $\mu$, GB: $7.20 \times 10^{3} / \mu 1$, neutrófilos: $50.8 \%$, linfocitos: $47.6 \%$, monocitos: $0.15 \%$, eosinófilos: $1.06 \%$, basófilos: $0.4 \%$, plaquetas: $227 \times 10^{3} / \mu 1, \mathrm{Hb}: 12.39 \mathrm{~g} / \mathrm{dl}, \mathrm{Ht}: 37.97 \%$ e índices hematológicos de VGM: 76.1 fl, HGM: 24.8 pg, CHGM: 32.6 g/dl. No se encontró diferencias significativas entre grupos etáreos ni entre sexos.
\end{abstract}

Palabras clave : machín negro, Cebus apella, hematología, cautiverio

\section{Abstract}

The objective of the present study was to determine referential hematological values of the tufted capuchin (Cebus apella) maintained in captivity at the Patronato del Parque de Las Leyendas, Lima. A total of 32 primates (25 males and 7 females), among juveniles, sub adults and adults, in apparent good conditions were used. The primates were i.m. anesthetized with a combination of ketamine $(10 \mathrm{mg} / \mathrm{kg} / \mathrm{bw})$ and xylazine $(1 \mathrm{mg} / \mathrm{kg} / \mathrm{bw})$. The samples were obtained by puncture of the femoral vein using vacutainers with

\footnotetext{
${ }^{1}$ Laboratorio de Patología Clínica, ${ }^{5}$ Clinica de Animales Menores, Facultad de Medicina Veterinaria, Universidad Nacional Mayor de San Marcos, Lima

${ }^{2}$ E-mail: pedrospinasal@hotmail.com

${ }^{3}$ E-mail: olgalie@hotmail.com

${ }^{4}$ Jefe de Patología, Patronato del Parque de las Leyendas (PATPAL), Lima
} 
EDTA as anticoagulant. The red blood cell (RBC) and white blood cell (WBC) count were performed using the chamber of Neubauer, the platelet count and differential using the Wright stain, the hemoglobin $(\mathrm{Hb})$ with the cianometahemoglobin method, the hematocrit (Ht) with the microhematocrit method and the eritrocitic indexes applying standard formulas with $\mathrm{RBC}, \mathrm{Hb}$ and $\mathrm{Ht}$ values. The average values were RBC: $5.12 \times 10^{6} / \mu 1, \mathrm{WBC}: 7.2$ x $10^{3} / \mu 1$, neutrophils: $50.8 \%$, lymphocytes: $47.6 \%$, monocytes: $0.15 \%$, eosinophils: $1.06 \%$,

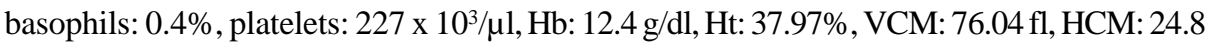
pg, CHCM: $32.6 \mathrm{~g} / \mathrm{dl}$. There were not significant differences due to age or sex.

Key words: tufted capuchin, Cebus apella, hematology, captivity

\section{INTRODUCCIÓN}

El Cebus apella, conocido en Perú como machín negro, es un primate de amplia distribución en la Amazonía peruana (Encarnación et al, 1993; Aquino y Encarnación, 1994), que viene siendo objeto de caza por los pobladores locales, ya que ven en ellos una fuente de alimento por su carne, y de ingreso económico por la venta de las crías (Emmons, 1999; Aquino et al., 2000). Este primate, por ser susceptible a enfermedades humanas, es utilizado como modelo experimental, siendo de gran importancia para la medicina y la salud humana (Hearn, 1983; Larsson et al., 1999).

La práctica de cazar para consumo y de comercializar las crías se está incrementando; por ello, se le observa comúnmente como mascota, no solo en poblados amazónicos, sino en centros urbanos de la costa, especialmente en Lima. El gran número de primates viviendo en cautiverio y como mascotas hace que el número de ellos en las clínicas veterinarias haya aumentado. Esto implica que el Médico Veterinario debe contar con valores referenciales hematológicos para evaluar el estado sanitario del paciente y poder realizar el diagnóstico apropiado.

El presente estudio tuvo como objetivo determinar los valores hematológicos referenciales de la cantidad de eritrocitos, hemoglobina, hematocrito, recuento plaquetario, índices eritrocíticos, cantidad de leucocitos y el recuento diferencial celular para el machín negro (Cebus apella) mantenido en cautive- rio, teniendo en cuenta el sexo y edad de los primates alojados en ambientes techados.

\section{Materiales y Métodos}

El estudio se realizó con animales del Patronato del Parque de Las Leyendas (PATPAL) de la ciudad de Lima, durante el mes de abril del 2004. El procesamiento de las muestras se llevó a cabo en el Laboratorio de Patología Clínica de la Facultad de Medicina Veterinaria de la Universidad Nacional Mayor de San Marcos, Lima.

Se utilizó toda la población de machín negro (Cebus apella), clínicamente sanos (25 machos y 7 hembras), agrupados por edad como juveniles (6 machos y 3 hembras), sub-adultos (12 machos y 4 hembras) y adultos (7 machos), según la tabla de Napier y Napier (1967) y los datos de las historias clínicas del PATPAL. Todos los animales se encontraban mantenidos bajo las mismas condiciones de alojamiento, alimentación, manejo y control sanitario.

En la mañana de la toma de muestra, los animales sometidos a ayuno previo, fueron atrapados con mallas en sus ambientes, pesados y anestesiados con una combinación de clorhidrato de ketamina $(10 \mathrm{mg} / \mathrm{kg} /$ pv) y xilazina $(1 \mathrm{mg} / \mathrm{kg} / \mathrm{pv})$ i.m. en el muslo. La toma de muestra de sangre se realizó por punción en la vena femoral, colectando la sangre en tubos vacutainer estériles con EDTA como anticoagulante. Los tubos fue- 
Cuadro 1. Valores de la serie eritrocítica y plaquetaria en machines negros (Cebus apella) mantenidos en cautiverio en el Parque de Las Leyendas $(n=32)$

\begin{tabular}{lcc}
\hline Variables & Promedio & Desviación estándar \\
\hline Eritrocitos $\left(\times 10^{6} / ? \mathrm{l}\right)$ & 5.12 & 0.88 \\
Hemoglobina $(\mathrm{g} / \mathrm{dl})$ & 12.39 & 0.98 \\
Hematocrito $(\%)$ & 37.97 & 2.55 \\
Plaquetas $\left(\times 10^{3} / ? 1\right)$ & 227.00 & 28.28 \\
$\mathrm{VCM}^{1}(\mathrm{fl})$ & 76.04 & 12.85 \\
$\mathrm{HCM}^{2}(\mathrm{pg})$ & 24.84 & 4.49 \\
$\mathrm{CHCM}^{3}(\mathrm{~g} / \mathrm{dl})$ & 32.63 & 1.43 \\
\hline${ }^{1}$ Volumen Corpuscular Medio; ${ }^{2}$ Hemoglobina Corpuscular Media, ${ }^{3}$ Concentración de Hemoglobina \\
Corpuscular Media
\end{tabular}

Cuadro 2. Valores de la serie leucocítica en machines negros (Cebus apella) mantenidos en cautiverio en el Parque de Las Leyendas $(n=32)$

\begin{tabular}{lcc}
\hline Variables & Promedio & Desviación estándar \\
\hline Leucocitos $(\mathrm{x} \mathrm{10} /$ /?1) & 7.20 & 2.02 \\
Neutrófilos (\%) & 50.75 & 13.37 \\
Linfocitos (\%) & 47.56 & 12.82 \\
Monocitos (\%) & 0.15 & -- \\
Eosinófilos (\%) & 1.06 & 1.5 \\
Basófilos (\%) & 0.40 & 0.53 \\
\hline
\end{tabular}

ron homogenizados, rotulados, almacenados a $4{ }^{\circ} \mathrm{C}$ y llevados al laboratorio para su procesamiento.

Se evaluaron los siguientes valores hematológicos:

- $\quad$ Eritrocitos $\left(\mathrm{x} 10^{6} / \mu \mathrm{l}\right)$ y leucocitos $\left(\mathrm{x} 10^{3 /}\right.$ $\mu \mathrm{l})$ utilizando la cámara de Neubauer.

- Hemoglobina (g/dl) por el método de la cianometahemoglobina.

- Hematocrito (\%) por el método del microhematocrito.

- Recuento diferencial (\%) y recuento plaquetario $(\mu \mathrm{l})$ utilizando la tinción Wright.
- Índices eritrocíticos: Volumen Corpuscular Medio (VCM), Hemoglobina Corpuscular Media (HCM) y Concentración de Hemoglobina Corpuscular Media (CHCM) en base al número de eritrocitos, cantidad de hemoglobina $\mathrm{y}$ hematocrito.

Los resultados se expresaron con la media aritmética como medida de tendencia central y la desviación estándar como medida de dispersión. Para hallar las diferencias de los parámetros hematológicos de la serie eritrocítica, serie leucocítica y plaquetas por efecto del sexo, se empleó la prueba de "t" de 
Cuadro 3. Valores de la serie eritrocítica y plaquetaria reportados en la literatura para el Machín Negro (Cebus apella)

\begin{tabular}{lccccc}
\hline Variables & $\begin{array}{c}\text { Ospina } \\
(2005)\end{array}$ & $\begin{array}{c}\text { ISIS } \\
(2004)\end{array}$ & $\begin{array}{c}\text { Larsson et al. } \\
(1999)\end{array}$ & $\begin{array}{c}\text { Almeida } \\
(1990)\end{array}$ & $\begin{array}{c}\text { Málaga et al. } \\
(1983)\end{array}$ \\
\hline $\begin{array}{l}\text { Eritrocitos } \\
\left(\mathrm{x} 10^{6} / ? \mathrm{l}\right)\end{array}$ & $5.12 ? 0.88$ & $5.88 ? 0.98$ & $5.4 ? 0.6$ & $4.75 ? 1.2$ & $5.45 ? 1.0$ \\
$\begin{array}{l}\text { Hemoglobina } \\
(\mathrm{g} / \mathrm{dl})\end{array}$ & $12.39 ? 0.98$ & $15.5 ? 10.4$ & $13.55 ? 1.5$ & $11.81 ? 1.3$ & $13.4 ? 1.9$ \\
$\begin{array}{l}\text { Hematocrito } \\
(\%)\end{array}$ & $37.97 ? 2.55$ & $42.4 ? 4.6$ & $42.5 ? 4.0$ & $35.44 ? 3.8$ & $39.9 ? 4.7$ \\
$\begin{array}{l}\text { Plaquetas } \\
\left(\mathrm{x} 10^{3} / ? 1\right)\end{array}$ & $227 ? 28.28$ & $408 ? 149$ & -- & -- & -- \\
$\mathrm{VCM}^{1}(\mathrm{fl})$ & $76.04 ? 12.85$ & $75 ? 8.4$ & $78.65 ? 8.1$ & $79.02 ? 18.2$ & $75.3 ? 13.4$ \\
$\mathrm{HCM}^{1}(\mathrm{pg})$ & $24.84 ? 4.49$ & $24.7 ? 2.2$ & $25.07 ? 3.2$ & $26.2 ? 6.2$ & $5.0 ? 3.1$ \\
$\mathrm{CHCM}^{1}(\mathrm{~g} / \mathrm{dl})$ & $32.63 ? 1.43$ & $33.2 ? 2.7$ & $31.7 ? 2.3$ & $33.11 ? 0.8$ & $33.6 ? 3.1$ \\
\hline
\end{tabular}

${ }^{1}$ Volumen Corpuscular Medio; ${ }^{2}$ Hemoglobina Corpuscular Media, ${ }^{3}$ Concentración de Hemoglobina Corpuscular Media

Cuadro 4. Valores de la serie leucocítica reportados en la literatura para el Machín Negro (Cebus apella)

\begin{tabular}{lccccc}
\hline Variables & $\begin{array}{c}\text { Ospina } \\
(2005)\end{array}$ & $\begin{array}{c}\text { ISIS } \\
(2004)\end{array}$ & $\begin{array}{c}\text { Larsson } \text { et al. } \\
(1999)\end{array}$ & $\begin{array}{c}\text { Almeida } \\
(1990)\end{array}$ & $\begin{array}{c}\text { Málaga } \text { et al. } \\
(1983)\end{array}$ \\
\hline $\begin{array}{l}\text { Leucocitos } \\
\left(\mathrm{x} 10^{6} / ? 1\right)\end{array}$ & $7.20 ? 2.0$ & $8.06 ? 3.7$ & $6.38 ? 3.1$ & $11.65 ? 3.3$ & $11.34 ? 5.7$ \\
$\begin{array}{l}\text { Neutrófilos } \\
(\%)\end{array}$ & $50.75 ? 13.4$ & $53.78 ? 3.5$ & $36.5 ? 2.7$ & $38.92 ? 16.0$ & $36.6 ? 16.5$ \\
$\begin{array}{l}\text { Linfocitos } \\
(\%)\end{array}$ & $47.56 ? 12.8$ & $27.96 ? 16.6$ & $27.96 ? 1.2$ & $57.5 ? 16.1$ & $50.4 ? 18.0$ \\
$\begin{array}{l}\text { Monocitos } \\
(\%)\end{array}$ & 0.2 & $0.32 ? 0.3$ & $0.18 ? 0.2$ & $1.06 ? 1.1$ & $4.5 ? 3.6$ \\
$\begin{array}{l}\text { Eosinófilos } \\
(\%)\end{array}$ & $1.06 ? 1.5$ & $0.22 ? 2.2$ & $0.035 ? 0.1$ & $1.54 ? 1.4$ & $7.2 ? 5.6$ \\
$\begin{array}{l}\text { Basófilos } \\
(\%)\end{array}$ & $0.4 ? 0.5$ & $0.10 ? 0.1$ & $0.03 ? 0.0$ & $0.64 ? 0.7$ & $1.5 ? 1.6$ \\
\hline
\end{tabular}


Student para muestras independientes y para hallar las diferencias por efecto de la edad se utilizó el análisis de varianza de una vía.

\section{Resultados}

Los valores hematológicos obtenidos en los machines negros se muestran en las Cuadros 1 y 2 . No se observó diferencias estadísticas significativas por efecto del sexo o la edad para la serie eritrocítica y la leucocítica.

\section{Discusión}

En el Perú se han realizado estudios similares sobre la hematología de los machines negros (Cebus apella) (Málaga et al., 1983; Almeida, 1990); así mismo, se han realizado trabajos con esta especie en zoológicos de Brasil (Larsson et al., 1999) y el International Species Information System (ISIS, 2004) ha recopilado datos provenientes de evaluaciones médicas periódicas de machines pertenecientes a diversos zoológicos del mundo.

Los resultados hallados en el presente estudio para la serie eritrocítica son similares a los hallados por otros autores (Cuadro 3). Para la hemoglobina, ISIS (2004) presenta el promedio más alto con una amplia dispersión. Según Medway et al. (1986), esta diferencia es probablemente debida al tipo de alimentación. Además, para esta prueba existen varias técnicas de evaluación como el método de la oxihemoglobina, la hematina ácida y de la cianometahemoglobina con un error estándar de 10,15 y $2 \%$, respectivamente.

Los valores de la serie trombocítica son menores a los encontrados en el ISIS (2004), variación que puede estar relacionada con la toma de la muestra o el estado sanitario de los primates al momento del muestreo. Asimismo, los valores de la serie leucocítica del presente estudio son similares a otros reportes de la literatura (Larsson et al., 1999; ISIS, 2004), o ligeramente inferiores (Málaga et al.,
1983; Almeida, 1990) (Cuadro 4). Esta diferencia podría deberse a que el conteo total de leucocitos está influenciado por el lugar de toma de la muestra, la actividad muscular y factores de estrés en el animal (Rebar et al., 2002).

Se encontró únicamente un monocito por animal en cinco primates (4 machos y 1 hembra), a diferencia de otros reportes (Málaga et al., 1983; Almeida, 1990), donde muestran valores más elevados. Este aumento puede estar influenciado por el estado sanitario de los primates, debido principalmente a la existencia de una infección subclínica (Rebar et al., 2002).

\section{Conclusiones}

- Se determinaron los valores hematológicos referenciales para el machín negro (Cebus apella) mantenidos en cautiverio.

- No se encontraron diferencias significativas en las series eritrocítica y leucocítica por efecto del sexo y la edad.

\section{Literatura Citada}

1. Almeyda H. 1990. Constantes hematológicas en primates en cautiverio de la especie Cebus apella en el Zoológico de San Miguel, Lima. Tesis de Médico Veterinario. Lima: Facultad de Medicina Veterinaria, Univ. Nacional Mayor de San Marcos. 49 p.

2. Aquino R, Encarnación F. 1994. Los primates del Perú. Germany: Ed. Golze. $90 \mathrm{p}$.

3. Aquino R, Bodmer R, Gil G. 2000. Impacto de la caza en poblaciones de primates de la cuenca del río Samiria, Reserva Nacional Pacaya Samiria. En: La primatología en el Perú. Vol. II. Lima: Facultad de Medicina Veterinaria, UNMSM. p 81-89. 
4. Emmons L. 1999. Mamíferos de los bosques húmedos de América tropical. Una guía de campo. Santa Cruz de la Sierra, Bolivia: Ed. F.N. 298 p.

5. Encarnación F, Moya L, Aquino R, Tapia J, Soini P. 1993. Situación y estado actual de las especies de primates no humanos en el Perú. En: La primatología en el Perú. 2000. Vol. II. Lima: Facultad de Medicina Veterinaria, UNMSM. p 219-228.

6. Hearn J. 1983. Reproduction in new world primates, new models in medical science. Lancaster: International Medical Publishers. 223 p.

7. [ISIS] International Species Information System. 2004. Clinical pathology records report. In house reference values mammals. American Units. [Internet]. Disponible en: http:// www.worldzoo.org/

8. Larsson M, Birgel EH, Benesi F, Birgel E, Lazaretti P, Fedullo J,
Larsson C, Molina S, Castro P, Prada

C. 1999. Hematological values of Cebus apella anesthetized with ketamine. Braz J Vet Res Anim Sci 36: 1413-1418.

9. Málaga C, Horna M, Villavicencio E, Moro J. 1983. Valores hemáticos normales del Saimiri boliviensis peruviensis y Cebus apella mantenidos en cautiverio en el centro de Reproducción y Conservación de Primates No Humanos (CRCP), Iquitos, Perú. En: La Primatología en el Perú. Lima: Facultad de Medicina Veterinaria, UNMSM. $p$ 565-570.

10. Medway W, Prior J, Wilkinson J. 1986. Patología clínica veterinaria. México: Ed Hispano-América. 532 p.

11. Napier J, Napier P. 1967. A handbook of living primates. New York: Academic Press London. $92 \mathrm{p}$.

12. Rebar A, MacWilliams P, Metzeger F. 2002. Manual de hematología de perros y gatos. Barcelona: Ed. Multimédica. 152 p. 